\title{
Going Forward with Lymphocytes Immunotherapies for Treatment of Unexplained Recurrent Spontaneous Abortion
}

\author{
Eloisa I Arana ${ }^{1,2}$ and Pablo M Fernández ${ }^{1,2 *}$ \\ ${ }^{1}$ Faculty of Pharmacy and Biochemistry, University of Buenos Aires, Argentina \\ ${ }^{2}$ Department of Immunology, University of Buenos Aires, Argentina, Email: pfernandez1120@gmail.com
}

*Corresponding author: Pablo M Fernández, Faculty of Pharmacy and Biochemistry, Department of Immunology, Hospital de Clínicas José de San Martín, Buenos Aires, Argentina.

To Cite This Article: Pablo M Fernández, Going Forward with Lymphocytes Immunotherapies for Treatment of Unexplained Recurrent Spontaneous Abortion. Am J Biomed Sci \& Res. 2019 - 6(5). AJBSR.MS.ID.001063. DOI: 10.34297/AJBSR.2019.06.001063.

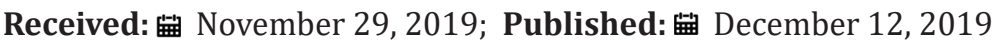

\section{Introduction}

Recurrent spontaneous abortion (RSA), defined as the occurrence of two or more consecutive miscarriages at less than 22-weeks of gestation [1], is one of the major pregnancy disorders and affects up to $5 \%$ of couples of reproductive ages. Although genetic factors, hormonal diseases, anatomical anomalies and antiphospholipid syndrome have been linked with RSA [2,3], 50\% of cases still remain unexplained and generate growing interest to unveil potential involved mechanisms, particularly for those that imply immunological pathways.

Successful pregnancy requires a highly regulated immune balance to achieve embryonic antigen tolerance. The embryo is considered a semi-homograft that is not rejected by the maternal immune system in a normal pregnancy [4,5]. Any disruption of this immune tolerance may have as a consequence pregnancy complication including spontaneous abortion.

The first report proposing an alloimmune mechanism as the cause of RSA was published in 1981 and suggested that similarities in human leukocyte antigens between father and mother would result in the failed production of blocking antibodies, thus leading to pregnancy termination [6]. The same year Taylor and Faulk published their results from treating three patients with unexplained RSA using allogeneic lymphocyte immunotherapy (LIT) [7], suggesting that infusion of allogeneic leukocytes stimulated the maternal immune system to produce immune tolerance factors that prevented spontaneous abortion.

Almost 40 years after these original reports, despite the extensive research in the field of reproductive immunology, there is a lack of robust scientific evidence concerning the use of immune therapies to treat RSA. Furthermore, results from the different studies evaluating immune therapies, including paternal (or third-party) LIT, but also other approaches like intravenous immunoglobulin (IVIG), or administration of trophoblast membranes, are contradictory.

Centers worldwide have been performing LIT as research projects, taking the rate of live birth in RSA patients as the main output. From these studies, a first meta-analysis published in 1993 by Fraser et al. evaluated the effect of LIT and did not detect any improvement in the rate of live births [8]. Shortly after, in 1994, a multicenter study and meta-analysis designed to assess the efficacy of LIT concluded that this therapy improved the rate of live births in RSA patients with no more than one live birth with any or current partner [9]. A significant improvement was observed when the patients had antibodies against the lymphocytes of their spouses before pregnancy. Similar results were reported by our research unit in 2000 [10].

A meta-analysis published by Cochrane Library in 2001 and updated in 2014 assessed different forms of immunologic treatment for RSA cases, including LIT, in a total of 641 participants (316 treated women and 325 in the placebo group) and showed that the treatment effect was not significant [11]. The analysis included the results presented by by Ober et al. in 1999, the only study published to date observing a negative effect of LIT on the rate of live births [12]. This was source of criticisms from researchers in the field, mainly because Ober et al. did not exclude patients with autoimmune disorders, but also prepared and used paternal lymphocytes in conditions that adversely impact in the results of 


\section{LIT [13-15].}

Recently, Liu et al. published a new meta-analysis including numerous clinical trials conducted from 1985 to 2013, and demonstrated that LIT promoted a significant improvement in the rate of live births $(77.8 \%$ in treated RSA patients compared to $46.1 \%$ in the control group), independently of the lymphocytes origin (paternal or third-party), and contributing to the understanding of the best protocol of immunization [16].

The latest works in the field confirm that LIT can be used to treat RSA patients to help preventing spontaneous abortion $[17,18]$, and explore predictor factors to reach better LIT outcomes $[19,20]$. Overall, current evidence demonstrates that LIT, when performed with fresh, non-stored blood, before and during pregnancy, with an adequate concentration of lymphocytes, and applied intradermally, is a valid and safe treatment for couples with history of RSA of unknown cause.

\section{References}

1. Zegers Hochschild F, Adamson GD, Dyer S, Racowsky C, de Mouzon J, et al. (2017) The International glossary on infertility and fertility care. Fertil Steril 108(3): 393-406.

2. Garrido Gimenez C, Alijotas Reig J (2015) Recurrent miscarriage: causes, evaluation and management. Postgrad Med J 91(1073): 151-162.

3. Bender Atik R, Christiansen OB, Elson J, Kolte A, Lewis S, et al. (2018) ESHRE guideline: recurrent pregnancy loss. Hum Reprod Open 2018(2): $1-13$.

4. Guleria I, Sayegh MH (2007) Maternal acceptance of the fetus: True human tolerance. J Immunol 178(6): 3345-3351.

5. Bansal AS, Bajardeen B, Thum MY (2012) The basis and value of currently used immunomodulatory therapies in recurrent miscarriage. J Reprod Immunol 93(1): 41-51.

6. Beer AE, Quebbeman JF, Ayers JW, Haines RF (1981) Major histocompatibility complex antigens, maternal and paternal immune responses, and chronic habitual abortions in humans. Am J Obstet Gynecol 141(8): 987-999.

7. Taylor C, Faulk WP (1981) Prevention of recurrent abortion with leucocyte transfusions. Lancet 2(8237): 68-70.

8. Fraser EJ, Grimes DA, Schultz KF (1993) Immunization as therapy for recurrent spontaneous abortion: a review and metaanalysis. Obstet Gynecol 82(5): 854-859.

9. Coulam CB, Clark DA, Collins J, Scott JR (1994) Worldwide collaborative observational study and meta-analysis on allogenic leukocyte immunotherapy for recurrent spontaneous abortion. Am J Reprod Immunol 32(2): 55-72.

10. Ramhorst R, Agriello E, Zittermann S, Pando M, Larriba J, et al. (2000) Is the Paternal Mononuclear Cells' Immunization a Successful Treatment for Recurrent Spontaneous Abortion? Am J Reprod Immunol 44(3): 129135.

11. Wong LF, Porter TF, Scott JR (2014) Immunotherapy for recurrent miscarriage. Cochrane Database Syst Rev (10): CD000112.

12. Ober C, Karrison T, Odem RB, Barnes RB, Branch DW, et al. (1999) Mononuclear-cell immunisation in prevention of recurrent miscarriages: a randomised trial. Lancet 354(9176): 365-369.

13. Pandey MK, Thakur S, Agrawal S (2004) Lymphocyte immunotherapy and its probable mechanism in the maintenance of pregnancy in women with recurrent spontaneous abortion. Arch Gynecol Obstet 269(3): 161172 .

14. Clark DA (2009) Cell-surface CD200 may predict efficacy of paternal mononuclear leukocyte immunotherapy in treatment of human recurrent pregnancy loss. Am J Reprod Immunol 61(1): 75-84.

15. Clark DA (2012) The end of evidence-based medicine? Inflammopharmacol 20(4): 187-193.

16. Liu Z, Xu H, Kang X, Wang T, He L, et al. (2016) Allogenic lymphocyte immunotherapy for unexplained recurrent spontaneous abortion: a meta-analysis. Am J Reprod Immunol 76(6): 443-453.

17. Chen JL, Yang JM, Huang YZ, Li Y (2016) Clinical observation of lymphocyte active immunotherapy in 380 patients with unexplained recurrent spontaneous abortion. Int Immunopharmacol 40: 347-350.

18. Sarno M, Cavalcante MB, Niag M, Pimentel K, Luz I, et al. (2019) Gestational and perinatal outcomes in recurrent miscarriages couples treated with lymphocyte immunotherapy. Eur J Obstet Gynecol Reprod Biol X 3: 100036.

19. Cavalcante MB, Sarno M, Niag M, Pimentel K, Luz I, et al. (2018) Lymphocyte immunotherapy for recurrent miscarriages: Predictors of therapeutic success. Am J Reprod Immunol 79(6): e12833.

20. Hajipour H, Nejabati HR, Latifi Z, Hamdi K, Bahrami asl Z, et al. (2018) Lymphocytes immunotherapy for preserving pregnancy: Mechanisms and Challenges. Am J Reprod Immunol 80(3): e12853. 